\title{
QUANTITATIVE ESTIMATES ON THE ENHANCED BINDING FOR THE PAULI-FIERZ OPERATOR
}

\author{
JEAN-MARIE BARBAROUX, HELMUT LINDE, AND SEMJON VUGALTER
}

\begin{abstract}
For a quantum particle interacting with a short-range potential, we estimate from below the shift of its binding threshold, which is due to the particle interaction with a quantized radiation field.
\end{abstract}

\section{INTRODUCTION}

Recently, the question of enhanced binding in nonrelativistic QED has been extensively studied in several publications [10, 9, 6, 3, 2, 4]. Dressing a charged particle with photons increases the ability of a potential to confine it. For the Pauli-Fierz operator which describes a nonrelativistic particle interacting with a radiation field, this effect was proved for small values of the fine structure constant $\alpha$, first under the simplifying assumption that the spin of the particle is absent [9], and later generalized to the case of a particle with spin [6, 3]. In [2], it was shown that the effect of the enhanced binding is asymptotically small in $\alpha$ in the sense that the binding threshold for the Pauli-Fierz operator tends to the binding threshold for the corresponding Schrödinger operator as $\alpha$ tends to zero. Some quantitative estimates on this effect were obtained in 4 where it was proved that the difference between the binding threshold for the Schrödinger operator and the corresponding Pauli-Fierz operator with spin zero is at least of the order $\alpha$. In the work at hand, using a different method, we prove similar results for the more general case of a particle with spin zero or one half. Notice that studying the enhanced binding effect in the case of a particle with spin requires recovering one more term of the energy's expansion in powers of $\alpha$ than in the spinless case.

Date: 10 august 2005.

The authors gratefully acknowledge financial support from the following institutions: The European Union through the IHP network "Analysis and Quantum" HPRN-CT-2002-00277 (JMB and SV), the French Ministery of Research through the ACI "jeunes chercheurs" (JMB), the Volkswagen Stiftung and DIPUC of the Pontificía Universidad Católica (HL), and the DFG grant WE 1964/2 (SV). 
The method of the proof is a further development of a method used in [9] and [6]. We prove that the Pauli-Fierz operator has a ground state even for some value of the potential coupling constant that is smaller than the binding threshold for the corresponding Schrödinger operator. To do so, we construct a trial function for which the quadratic form of the Pauli-Fierz operator with this coupling constant takes a value strictly less than the self-energy. Then we apply [8, Theorem 2.1] which tells us that this implies the existence of a ground state. The trial function we use is similar to the one in [6] with some modifications necessary to obtain quantitative estimates in the case with spin. It is constructed using the ground state of the self-energy operator with total momentum zero.

As in all previous papers [9, 6, 3, 2, 4, our method is asymptotic in $\alpha$. Therefore, the problem of establishing the enhanced binding effect and estimating its strength for the physical value of $\alpha \approx 1 / 137$ still remains open.

\section{Definitions AND MAIN RESUlt}

The Pauli-Fierz Hamiltonian $H$ for a charged particle with or without spin in an external electrostatic potential and coupled to the quantized electromagnetic radiation field is defined by

$H=\left(-i \nabla_{x} \otimes I_{f}+\sqrt{\alpha} A(x)\right)^{2}+g \sqrt{\alpha} \sigma \cdot B(x)+\lambda W(x) \otimes I_{f}+I_{e l} \otimes H_{f}-c_{\text {n.o. }} \alpha$.

The operator $H$ acts on the Hilbert space $\mathfrak{H}:=\mathfrak{H}^{e l} \otimes \mathfrak{F}$. The Hilbert space $\mathfrak{H}^{e l}$ of the nonrelativistic particle is $L^{2}\left(\mathbb{R}^{3}\right) \otimes \mathbb{C}^{2}$ in the case $g=1$ and $L^{2}\left(\mathbb{R}^{3}\right)$ in the case $g=0$. Here $\mathbb{R}^{3}$ is the configuration space of a single particle, while $\mathbb{C}^{2}$ accommodates its spin in the case $g=1$.

We will describe the quantized electromagnetic field by use of the Coulomb gauge condition. Accordingly, the one-photon Hilbert space is given by $L^{2}\left(\mathbb{R}^{3}\right) \otimes \mathbb{C}^{2}$, where $\mathbb{R}^{3}$ denotes either the photon momentum or configuration space, and $\mathbb{C}^{2}$ accounts for the two independent transversal polarizations of the photon. The photon Fock space is then defined by

$$
\mathfrak{F}=\bigoplus_{n=0}^{\infty} \mathfrak{F}_{s}^{(n)}
$$

where the n-photons space $\mathfrak{F}_{s}^{(n)}=\bigotimes_{s}^{n}\left(L^{2}\left(\mathbb{R}^{3}\right) \otimes \mathbb{C}^{2}\right)$ is the symmetric tensor product of $n$ copies of $L^{2}\left(\mathbb{R}^{3}\right) \otimes \mathbb{C}^{2}$. 
We use units such that $\hbar=c=1$, and where the mass of the particle equals $m=1 / 2$. The particle charge is then given by $e=\sqrt{\alpha}$. As usual, we will consider $\alpha$ as a small parameter.

The operator that couples a particle to the quantized vector potential is given by

$$
\begin{aligned}
A(x) & =\sum_{\lambda=1,2} \int_{\mathbb{R}^{3}} \frac{\zeta(|k|)}{2 \pi|k|^{1 / 2}} \varepsilon_{\lambda}(k)\left[e^{i k x} \otimes a_{\lambda}(k)+e^{-i k x} \otimes a_{\lambda}^{*}(k)\right] \mathrm{d} k \\
& =: D(x)+D^{*}(x),
\end{aligned}
$$

where $\operatorname{div} A=0$ by the Coulomb gauge condition. The operators $a_{\lambda}$, $a_{\lambda}^{*}$ satisfy the usual commutation relations

$$
\left[a_{\nu}(k), a_{\lambda}^{*}\left(k^{\prime}\right)\right]=\delta\left(k-k^{\prime}\right) \delta_{\lambda, \nu}, \quad\left[a_{\nu}(k), a_{\lambda}\left(k^{\prime}\right)\right]=0 .
$$

The vectors $\varepsilon_{\lambda}(k) \in \mathbb{R}^{3}$ are the two orthonormal polarization vectors perpendicular to $k$,

$$
\varepsilon_{1}(k)=\frac{\left(k_{2},-k_{1}, 0\right)}{\sqrt{k_{1}^{2}+k_{2}^{2}}} \quad \text { and } \quad \varepsilon_{2}(k)=\frac{k}{|k|} \wedge \varepsilon_{1}(k) .
$$

The function $\zeta(|k|)$ describes the ultraviolet cutoff on the wavenumbers $k$. We assume $\zeta$ to be of class $C^{1}$ and to have compact support.

The constant $c_{\text {n.o. }}$ is

$$
c_{\text {n.o. }}=\left[D, D^{*}\right]=\frac{2}{\pi} \int_{0}^{\infty} r|\zeta(r)|^{2} \mathrm{~d} r
$$

and subtraction of the constant $c_{\text {n.o. }} \alpha$ amounts to normal ordering of the operator $A^{2}$.

The operator that couples a particle to the magnetic field $B=\operatorname{curl} A$ is given by

$$
\begin{aligned}
B(x) & =\sum_{\lambda=1,2} \int_{\mathbb{R}^{3}} \frac{\zeta(|k|)}{2 \pi|k|^{1 / 2}} k \times i \varepsilon_{\lambda}(k)\left[e^{i k x} \otimes a_{\lambda}(k)-e^{-i k x} \otimes a_{\lambda}^{*}(k)\right] \mathrm{d} k \\
& =: K(x)+K^{*}(x) .
\end{aligned}
$$

In Equation (11), $\sigma=\left(\sigma_{1}, \sigma_{2}, \sigma_{3}\right)$ is the 3-component vector of Pauli matrices

$$
\sigma_{1}=\left(\begin{array}{ll}
0 & 1 \\
1 & 0
\end{array}\right), \quad \sigma_{2}=\left(\begin{array}{cc}
0 & -i \\
i & 0
\end{array}\right), \quad \sigma_{3}=\left(\begin{array}{cc}
1 & 0 \\
0 & -1
\end{array}\right) .
$$

The photon field energy operator $H_{f}$ is given by

$$
H_{f}=\sum_{\lambda=1,2} \int_{\mathbb{R}^{3}}|k| a_{\lambda}^{*}(k) a_{\lambda}(k) \mathrm{d} k .
$$


The multiplicative potential $W$ is assumed to be short range and in $L_{\text {loc }}^{4}\left(\mathbb{R}^{3}\right)$, and $\lambda$ is a positive coupling constant. If the negative part of $W$ is nontrivial, then there exists a critical value $\lambda_{0}$ such that the Schrödinger operator $-\Delta+\lambda W$ has discrete spectrum for all $\lambda>\lambda_{0}$, but does not have any discrete spectrum for $0 \leq \lambda<\lambda_{0}$. Analogously, the Pauli-Fierz operator also has a critical coupling constant $\lambda_{1}$, which depends on the fine structure constant $\alpha$. It is known [2] that $\lambda_{1}$ converges to $\lambda_{0}$ from below as $\alpha$ goes to zero.

Before stating our main result, let us introduce some notations. For $v$ a measurable function in $\mathbb{R}^{3}$, we define

$$
d_{v}=\frac{1}{2 \pi}\left(\int \frac{|v(x)| \mid v(y \mid}{|x-y|^{2}} \mathrm{~d} x \mathrm{~d} y\right)^{\frac{1}{2}}
$$

if $v$ is not spherically symmetric and

$$
d_{v}=\min \left\{\frac{1}{2 \pi}\left(\int \frac{|v(x)| \mid v(y \mid}{|x-y|^{2}} \mathrm{~d} x \mathrm{~d} y\right)^{\frac{1}{2}}, \int_{0}^{\infty} t|v(t)| \mathrm{d} t\right\}
$$

if $v$ is spherically symmetric.

Our main result is thus

Theorem 2.1. Assume that $W(x)$ satisfies the following conditions: $W \in L_{\text {loc }}^{4}\left(\mathbb{R}^{3}\right)$ and there exists $a>0, c>0$ and $\delta>0$ such that for all $|x|>a,|W(x)| \leq c(1+|x|)^{-2-\delta}$. Then

$$
\lambda_{1} \leq \lambda_{0}\left(1-\alpha \eta^{2}+\mathcal{O}\left(\alpha^{\frac{5}{4}}\right)\right)
$$

with

$$
\eta^{2}=\frac{1}{6 \pi^{2}} \int_{\mathbb{R}^{3}} \frac{\zeta(|k|)}{|k|\left(k^{2}+|k|+C_{W}\right)} \mathrm{d} k
$$

and

$$
C_{W}=\lambda_{0}^{2}\left(1+\lambda_{0} d_{W_{+}}\right) d_{W^{2}} \quad \text { and } \quad W_{+}=(|W|+W) / 2 .
$$

\section{Proof of the Main Theorem}

In this section, we will prove the main theorem in the case of particle with spin $g=1$. The proof for $g=0$ can easily be deduced with several simplifications.

We start with establishing some useful preliminary estimates.

\subsection{Properties of the self-energy operator $\mathbf{T}(0)$ with zero total} momentum. This section addresses the main properties of the selfenergy operator $T(0)$. Let us consider the case of a free particle coupled 
to the quantized electromagnetic field. The self-energy operator $T$ is given by

$$
T=\left(-i \nabla_{x} \otimes I_{f}+\sqrt{\alpha} A(x)\right)^{2}+g \sqrt{\alpha} \sigma \cdot B(x)+I_{e l} \otimes H_{f}-c_{\text {n.o. }} \alpha .
$$

We note that this system is translationally invariant, that is, $T$ commutes with the operator of total momentum

$$
P_{t o t}=p_{e l} \otimes I_{f}+I_{e l} \otimes P_{f},
$$

where $p_{e l}$ and $P_{f}=\sum_{\lambda=1,2} \int k a_{\lambda}^{*}(k) a_{\lambda}(k) \mathrm{d} k$ denote the particle and the photon momentum operators.

Let $\mathfrak{H}_{P} \cong \mathbb{C}^{2} \otimes \mathfrak{F}$ denotes the fibre Hilbert space corresponding to conserved total momentum $P$. For any fixed value $P$ of the total momentum, the restriction of $T$ to the fibre space $\mathfrak{H}_{P}$ is given by (see e.g. [5])

$$
T(P)=\left(P-P_{f}+\sqrt{\alpha} A(0)\right)^{2}+g \sqrt{\alpha} \sigma \cdot B(0)+H_{f}-c_{\text {n.o. }} \alpha .
$$

We denote $\Sigma_{0}:=\inf \sigma(T(0))$.

For the reader convenience, we first collect in the following theorem different known facts regarding the ground state of the operator $T(0)$, which will be used in the proof of the main theorem.

From now on, we will denote by $\Pi_{n}$ the projection onto the subspace of $\mathbb{C}^{2} \otimes \mathfrak{F}$ corresponding to vectors which have all components zero except the $n$-photon components. We also define $\Pi_{n}^{\geq}=1-\sum_{i=1}^{n-1} \Pi_{n}$.

For vectors in $\mathbb{C}^{2} \otimes \mathfrak{F}$, the norm $\|$.$\| will refer to the standard norm$ in $\mathbb{C}^{2} \otimes \mathfrak{F}$.

Theorem 3.1. 7, 5, 6, 1] For $\alpha$ sufficiently small we have:

- $\Sigma_{0}$ is an eigenvalue bordering to continuous spectrum of $T(0)$ and $\Sigma_{0}=\inf \sigma(T)$.

- For any $\Omega_{0} \in \operatorname{Ker}\left(T(0)-\Sigma_{0}\right)$, its projection $\Pi_{0} \Omega_{0}$ onto the zerophoton sector of $\mathbb{C}^{2} \otimes \mathfrak{F}$ fulfils $\left\|\Pi_{0} \Omega_{0}\right\| \neq 0$. If $\Omega_{0}$ is normalized by $\left\|\Pi_{0} \Omega_{0}\right\|=1$, then the following inequalities are satisfied: $\left\|\Omega_{0}\right\|=1+\mathcal{O}\left(\alpha^{1 / 2}\right),\left\|D(0) \Omega_{0}\right\|=\mathcal{O}\left(\alpha^{1 / 2}\right)$, and $\left\|H_{f}^{1 / 2} \Omega_{0}\right\|=$ $\mathcal{O}\left(\alpha^{1 / 2}\right)$.

- For the photon number operator $N_{f}:=\sum_{\lambda=1,2} \int a_{\lambda}^{*}(k) a_{\lambda}(k) \mathrm{d} k$, we have $\left\|N_{f}^{1 / 2} \Omega_{0}\right\|=\mathcal{O}\left(\alpha^{1 / 2}\right)$.

Corollary 3.2. For any vector $\Omega_{0} \in \operatorname{Ker}\left(T(0)-\Sigma_{0}\right)$ normalized by $\left\|\Pi_{0} \Omega_{0}\right\|=1$, we have $\left\|\Omega_{0}\right\|=1+\mathcal{O}(\alpha),\left\|D^{*}(0) \Pi_{1}^{\geq} \Omega_{0}\right\|=\mathcal{O}\left(\alpha^{1 / 2}\right)$ and $\left\|\sigma \cdot K^{*}(0) \Pi_{1}^{\geq} \Omega_{0}\right\|=\mathcal{O}\left(\alpha^{1 / 2}\right)$.

In the following, we consider two 4 -vectors in $\mathbb{C}^{2} \otimes\left(L^{2}\left(\mathbb{R}^{3}\right) \otimes \mathbb{C}^{2}\right)$, of the form $\left(\xi\left(\uparrow, k, \lambda_{1}\right), \xi\left(\uparrow, k, \lambda_{2}\right), \xi\left(\downarrow, k, \lambda_{1}\right), \xi\left(\downarrow, k, \lambda_{2}\right)\right)$, where $\uparrow$ and $\downarrow$ 
refer to the spin up and spin down of the particle, and $\lambda_{1}, \lambda_{2}$ refer to the two polarizations of the transverse photons.

$$
\Gamma_{a, b}:=\left(\begin{array}{l}
\Gamma\left(\uparrow, k, \lambda_{1}\right) \\
\Gamma\left(\uparrow, k, \lambda_{2}\right) \\
\Gamma\left(\downarrow, k, \lambda_{1}\right) \\
\Gamma\left(\downarrow, k, \lambda_{2}\right)
\end{array}\right):=\left(\begin{array}{l}
\frac{\zeta_{\Lambda}(k)}{|k|^{\frac{1}{2}}}\left(-a \sqrt{k_{1}^{2}+k_{2}^{2}}+b \frac{\left(k_{1}-i k_{2}\right) k_{3}}{\sqrt{k_{1}^{2}+k_{2}^{2}}}\right) \\
b \zeta_{\Lambda}(k) \frac{-k_{2}-i k_{1}}{\sqrt{k_{1}^{2}+k_{2}^{2}}|k|^{\frac{1}{2}}} \\
\frac{\zeta_{\Lambda}(k)}{|k|^{\frac{1}{2}}}\left(b \sqrt{k_{1}^{2}+k_{2}^{2}}+a \frac{\left(k_{1}+i k_{2}\right) k_{3}}{\sqrt{k_{1}^{2}+k_{2}^{2}}}\right) \\
a \zeta_{\Lambda}(k) \frac{-k_{2}+i k_{1}}{\sqrt{k_{1}^{2}+k_{2}^{2}}}|k|^{\frac{1}{2}}
\end{array}\right)
$$

Let

$$
\varphi_{a, b}=\sqrt{\alpha} \frac{i}{2 \pi|k|(1+|k|)} \Gamma_{a, b}
$$

Proposition 3.3 (Approximate ground state of the Pauli-Fierz operator). For $a$ and $b$ in $\mathbb{C}$ such that $|a|^{2}+|b|^{2}=1$, we consider the family of real-valued functionals $L_{a, b}$ defined on $\mathbb{C}^{2} \otimes L^{2}\left(\mathbb{R}^{3}\right) \otimes \mathbb{C}^{2}$ by

$$
L_{a, b}(\xi)=\left\langle\left(k^{2}+|k|\right) \xi, \xi\right\rangle+2 \sqrt{\alpha} \mathcal{R} e\left\langle\xi, \Pi_{1} \sigma \cdot K^{*}(0)\left(\left(\begin{array}{c}
a \\
b
\end{array}\right) 0,0, \cdots\right)\right\rangle
$$

where as before $B(0)=K(0)+K^{*}(0)$. Then we have

i) The vector $\varphi_{a, b}$ defined by (6) is the unique minimizer of $L_{a, b}$.

ii) $\left|\Sigma_{0}-\inf L_{a, b}(\xi)\right|=\mathcal{O}\left(\alpha^{3 / 2}\right)$.

iii) Let $\Omega_{0} \in \operatorname{Ker}\left(T(0)-\Sigma_{0}\right)$ be normalized by $\left\|\Pi_{0} \Omega_{0}\right\|=1$. Let us denote by $(a, b):=\Pi_{0} \Omega_{0}$. We define the scalar product $\langle., .\rangle_{1}$ onto the one-photon sector $\Pi_{1}\left(\mathbb{C}^{2} \otimes \mathfrak{F}\right)=\mathbb{C}^{2} \otimes L^{2}\left(\mathbb{R}^{3}\right) \otimes \mathbb{C}^{2}$ by $\langle f, g\rangle_{1}=$ $\left\langle\left(k^{2}+|k|\right) f, g\right\rangle_{\Pi_{1}\left(\mathbb{C}^{2} \otimes \mathfrak{F}\right)}$. Then for $\gamma \in \mathbb{R}$ and $R \in \mathbb{C}^{2} \otimes L^{2}\left(\mathbb{R}^{3}\right) \otimes \mathbb{C}^{2}$ such that

$$
\Pi_{1} \Omega_{0}=\gamma \varphi_{a, b}+R
$$

and $\left\langle\varphi_{a, b}, R\right\rangle_{1}=0$, we have

$$
\langle R, R\rangle_{1}=\mathcal{O}\left(\alpha^{3 / 2}\right) \text { and }|\gamma-1|=\mathcal{O}\left(\alpha^{3 / 4}\right)
$$

Remark 3.4. In the above proposition, and in the sequel, we use the same notation for $\Pi_{1} \Omega_{0}$ as a vector in $\mathbb{C}^{2} \otimes \mathfrak{F}$ which has all components zero except its one-photon component $\left(\Pi_{1} \Omega_{0}\right)^{(1)}$, as well as for the vector $\left(\Pi_{1} \Omega_{0}\right)^{(1)}$ in $\mathbb{C}^{2} \otimes L^{2}\left(\mathbb{R}^{3}\right) \otimes \mathbb{C}^{2}$.

Proof. In this proof, for the sake of simplicity of notations, we will drop the argument 0 in the operators $A(0), B(0), D(0), K(0)$ and their adjoint. We first prove i). Denoting

$$
g_{a, b}:=\frac{1}{\left(k^{2}+|k|\right)} \Pi_{1} \sigma \cdot K^{*}\left(\left(\begin{array}{l}
a \\
b
\end{array}\right), 0,0, \cdots\right),
$$


we have

$$
L_{a, b}(\xi)=\langle\xi, \xi\rangle_{1}+2 \sqrt{\alpha} \mathcal{R} e\left\langle\xi, g_{a, b}\right\rangle_{1}=\left\|\xi+\sqrt{\alpha} g_{a, b}\right\|_{1}^{2}-\left\|\sqrt{\alpha} g_{a, b}\right\|_{1}^{2},
$$

where $\|.\|_{1}$ is the norm associated to the scalar product $\langle., .\rangle_{1}$. Therefore, the minimizer of $L_{a, b}$ is $-\sqrt{\alpha} g_{a, b}$. A straightforward computation shows that $-\sqrt{\alpha} g_{a, b}=\varphi_{a, b}$. This implies that

$$
\inf L_{a, b}=L_{a, b}\left(\varphi_{a, b}\right)=-\left\|\varphi_{a, b}\right\|_{1}^{2} .
$$

We now prove ii). We have

$$
\begin{aligned}
\left\langle T(0) \Omega_{0}, \Omega_{0}\right\rangle= & \left\langle P_{f}^{2} \Omega_{0}, \Omega_{0}\right\rangle-\sqrt{\alpha} 2 \mathcal{R} e\left\langle P_{f} \Omega_{0}, A \Omega_{0}\right\rangle+\alpha\left\langle A^{2} \Omega_{0}, \Omega_{0}\right\rangle \\
& +\sqrt{\alpha}\left\langle\sigma \cdot B \Omega_{0}, \Omega_{0}\right\rangle+\left\langle H_{f} \Omega_{0}, \Omega_{0}\right\rangle-c_{\text {n.o. }} \alpha
\end{aligned}
$$

Let us estimate the terms in the above equality in order to identify those who are of order $\alpha^{3 / 2}$ and higher.

$$
\begin{aligned}
\left\langle P_{f}^{2} \Omega_{0}, \Omega_{0}\right\rangle & =\left\langle P_{f}^{2} \Pi_{0} \Omega_{0}, \Pi_{0} \Omega_{0}\right\rangle+\left\langle P_{f}^{2} \Pi_{1} \Omega_{0}, \Pi_{1} \Omega_{0}\right\rangle+\left\langle P_{f}^{2} \Pi_{2}^{\geq} \Omega_{0}, \Pi_{2}^{\geq} \Omega_{0}\right\rangle \\
& =\left\langle P_{f}^{2} \Pi_{1} \Omega_{0}, \Pi_{1} \Omega_{0}\right\rangle+\left\langle P_{f}^{2} \Pi_{2}^{\geq} \Omega_{0}, \Pi_{2}^{\geq} \Omega_{0}\right\rangle .
\end{aligned}
$$

$$
\begin{aligned}
\left\langle H_{f} \Omega_{0}, \Omega_{0}\right\rangle & =\left\langle H_{f} \Pi_{0} \Omega_{0}, \Pi_{0} \Omega_{0}\right\rangle+\left\langle H_{f} \Pi_{1} \Omega_{0}, \Pi_{1} \Omega_{0}\right\rangle+\left\langle H_{f} \Pi_{2}^{\geq} \Omega_{0}, \Pi_{2}^{\geq} \Omega_{0}\right\rangle \\
& =\left\langle H_{f} \Pi_{1} \Omega_{0}, \Pi_{1} \Omega_{0}\right\rangle+\left\langle H_{f} \Pi_{2}^{\geq} \Omega_{0}, \Pi_{2}^{\geq} \Omega_{0}\right\rangle .
\end{aligned}
$$

Now, using the fact that $n$-photon sectors are invariant under $P_{f}$, $P_{f} \Pi_{0} \Omega_{0}=0$, and $\left\langle P_{f} \Omega_{0}, A \Omega_{0}\right\rangle=\left\langle A P_{f} \Pi_{1}^{\geq} \Omega_{0}, \Omega_{0}\right\rangle=\left\langle A \Pi_{1}^{\geq} \Omega_{0}, P_{f} \Omega_{0}\right\rangle=$ $\left\langle A \Pi_{1}^{\geq} \Omega_{0}, P_{f} \Pi_{1}^{\geq} \Omega_{0}\right\rangle$, we get

$$
\begin{aligned}
\left|\left\langle P_{f} \Omega_{0}, A \Omega_{0}\right\rangle\right|= & \left|\left\langle P_{f} \Pi_{1}^{\geq} \Omega_{0}, A \Pi_{1}^{\geq} \Omega_{0}\right\rangle\right| \\
\leq & \left|\left\langle P_{f} \Pi_{1}^{\geq} \Omega_{0}, D \Pi_{2}^{\geq} \Omega_{0}\right\rangle\right|+\left|\left\langle P_{f} \Pi_{2}^{\geq} \Omega_{0}, D^{*} \Pi_{1}^{\geq} \Omega_{0}\right\rangle\right| \\
\leq & \left|\left\langle P_{f} \Pi_{1} \Omega_{0}, D \Pi_{2} \Omega_{0}\right\rangle\right|+\left|\left\langle P_{f} \Pi_{2}^{\geq} \Omega_{0}, D \Pi_{3}^{\geq} \Omega_{0}\right\rangle\right| \\
& +\left|\left\langle P_{f} \Pi_{2}^{\geq} \Omega_{0}, D^{*} \Pi_{1}^{\geq} \Omega_{0}\right\rangle\right| \\
\leq & \left\|P_{f} \Pi_{1} \Omega_{0}\right\|\left\|D \Pi_{2} \Omega_{0}\right\|+\frac{1}{2}\left\|P_{f} \Pi_{2}^{\geq} \Omega_{0}\right\|^{2}+ \\
& 2\left\|D \Pi_{3}^{\geq} \Omega_{0}\right\|^{2}+2\left\|D^{*} \Pi_{1}^{\geq} \Omega_{0}\right\|^{2} .
\end{aligned}
$$

Using Theorem 3.1 and Corollary 3.2 and the fact that $\left\|P_{f} \Pi_{1} \Omega_{0}\right\| \leq$ $c(\Lambda)\left\|\Pi_{1} \Omega_{0}\right\|=\mathcal{O}\left(\alpha^{1 / 2}\right)$, where $c(\Lambda)$ depends only on the ultraviolet cutoff, yields

$$
\left|\left\langle P_{f} \Omega_{0}, A \Omega_{0}\right\rangle\right| \leq \frac{1}{2}\left\|P_{f} \Pi_{2}^{\geq} \Omega_{0}\right\|^{2}+\mathcal{O}(\alpha) .
$$


We also have

$$
\begin{aligned}
& \left\langle A^{2} \Omega_{0}, \Omega_{0}\right\rangle \\
& =\left\langle\left(D+D^{*}\right)^{2} \Omega_{0}, \Omega_{0}\right\rangle \\
& =2 \mathcal{R} e\left\langle D D \Omega_{0}, \Omega_{0}\right\rangle+2\left\|D \Omega_{0}\right\|^{2}+\left\|\left[D, D^{*}\right]\right\|\left\|\Omega_{0}\right\|^{2} \\
& =\mathcal{O}\left(\alpha^{1 / 2}\right)+\mathcal{O}(\alpha)+\left\|\left[D, D^{*}\right]\right\|(1+\mathcal{O}(\alpha)),
\end{aligned}
$$

where we used from Theorem 3.1 that $\left\|\Omega_{0}\right\|=1+\mathcal{O}(\alpha)$ and $\left\|D \Omega_{0}\right\|=$ $\mathcal{O}\left(\alpha^{1 / 2}\right)$. Since the commutator $\left[D, D^{*}\right]$ equals $c_{\text {n.o. }}$ we arrive at

$$
\left\langle A^{2} \Omega_{0}, \Omega_{0}\right\rangle=c_{\text {n.o. }}+\mathcal{O}\left(\alpha^{1 / 2}\right) .
$$

Finally we have, writing $B=K+K^{*}$

$$
\begin{array}{r}
\left\langle\sigma \cdot B \Omega_{0}, \Omega_{0}\right\rangle=\left\langle\sigma \cdot K \Pi_{1} \Omega_{0}, \Pi_{0} \Omega_{0}\right\rangle+\left\langle\sigma \cdot K \Pi_{2}^{\geq} \Omega_{0}, \Pi_{1}^{\geq} \Omega_{0}\right\rangle \\
+\left\langle\sigma \cdot K^{*} \Pi_{0} \Omega_{0}, \Pi_{1} \Omega_{0}\right\rangle+\left\langle\sigma \cdot K^{*} \Pi_{1}^{\geq} \Omega_{0}, \Pi_{2}^{\geq} \Omega_{0}\right\rangle \\
=2 \mathcal{R} e\left\langle\sigma \cdot K \Pi_{1} \Omega_{0}, \Pi_{0} \Omega_{0}\right\rangle+2 \mathcal{R} e\left\langle\sigma \cdot K^{*} \Pi_{1}^{\geq} \Omega_{0}, \Pi_{2}^{\geq} \Omega_{0}\right\rangle .
\end{array}
$$

using Theorem 3.1 and Corollary 3.2 we obtain

$$
\left\langle\sigma \cdot B \Omega_{0}, \Omega_{0}\right\rangle=2 \mathcal{R} e\left\langle\sigma \cdot K \Pi_{1} \Omega_{0}, \Pi_{0} \Omega_{0}\right\rangle+\mathcal{O}(\alpha) .
$$

Collecting (10)-(15) and using $\left\langle H_{f} \Pi_{2}^{\geq} \Omega_{0}, \Pi_{2}^{\geq} \Omega_{0}\right\rangle \geq 0$ we obtain

$$
\begin{aligned}
\left\langle T(0) \Omega_{0}, \Omega_{0}\right\rangle & \geq\left\langle P_{f}^{2} \Pi_{1} \Omega_{0}, \Pi_{1} \Omega_{0}\right\rangle+\left\langle H_{f} \Pi_{1} \Omega_{0}, \Pi_{1} \Omega_{0}\right\rangle \\
& +2 \sqrt{\alpha} \mathcal{R} e\left\langle\sigma \cdot K \Pi_{1} \Omega_{0}, \Pi_{0} \Omega_{0}\right\rangle+\mathcal{O}\left(\alpha^{\frac{3}{2}}\right) .
\end{aligned}
$$

Since on the one-photon sector the operator $P_{f}^{2}$ reduces to multiplication by $k^{2}$, and the operator $H_{f}$ reduced to multiplication by $|k|$, we obtain

$$
\Sigma_{0}=\frac{\left\langle T(0) \Omega_{0}, \Omega_{0}\right\rangle}{\left\|\Omega_{0}\right\|^{2}} \geq L_{a, b}\left(\Pi_{1} \Omega_{0}\right)+\mathcal{O}\left(\alpha^{3 / 2}\right) \geq \inf L_{a, b}+\mathcal{O}\left(\alpha^{3 / 2}\right)
$$

On the other hand, using i), and for $\psi_{a, b}=\left(\left(\begin{array}{c}a \\ b\end{array}\right), \varphi_{a, b}, 0,0, \cdots\right)$ we have

$$
\begin{aligned}
\inf L_{a, b} & =L_{a, b}\left(\varphi_{a, b}\right)=\left\langle T(0) \psi_{a, b}, \psi_{a, b}\right\rangle \geq \Sigma_{0}\left\|\psi_{a, b}\right\|^{2} \\
& =\Sigma_{0}(1+\mathcal{O}(\alpha)) \geq \Sigma_{0}+\mathcal{O}\left(\alpha^{2}\right)
\end{aligned}
$$

Inequalities (17) and (18) conclude the proof of ii).

Eventually, we prove iii). Due to the Inequalities (17) and (18), we have $\inf L_{a, b}+\mathcal{O}\left(\alpha^{3 / 2}\right)=L_{a, b}\left(\Pi_{1} \Omega_{0}\right)$. Using (8), the fact that $-\sqrt{\alpha} g_{a, b}=\varphi_{a, b}$, and (9), we thus get

$$
\begin{aligned}
\inf L_{a, b}+\mathcal{O}\left(\alpha^{3 / 2}\right) & =L_{a, b}\left(\Pi_{1} \Omega_{0}\right)=\left\|\gamma \varphi_{a, b}+R-\varphi_{a, b}\right\|_{1}^{2}-\left\|\varphi_{a, b}\right\|_{1}^{2} \\
& =(\gamma-1)^{2}\left\|\varphi_{a, b}\right\|_{1}^{2}+\|R\|_{1}^{2}+\inf L_{a, b},
\end{aligned}
$$


which proves (17).

3.2. Proof of Theorem 2.1. As it was mentioned in the introduction, we prove the theorem by constructing a trial function $\Psi$ for which the quadratic form of $H$ takes a value strictly smaller than $\Sigma_{0}\|\Psi\|^{2}$.

Let us start by proving an auxiliary result. For $\gamma \in(0,1)$, we define $f_{\gamma} \in L^{2}\left(\mathbb{R}^{3}\right)$ to be a normalized real valued eigenfunction, with associated eigenvalue $e_{\gamma}$, of the Schrödinger operator

$$
h_{\gamma}:=-(1-\gamma) \Delta+\lambda_{0} W(x) .
$$

Here $\lambda_{0}$ is the critical coupling constant defined in Section 2.

Lemma 3.5. Then for $\lambda \leq \lambda_{0}$, we have

$$
\sum_{i}\left\langle(-\Delta+\lambda W) \frac{\partial f_{\gamma}}{\partial x_{i}}, \frac{\partial f_{\gamma}}{\partial x_{i}}\right\rangle \leq C_{W}\left\|\nabla f_{\gamma}\right\|^{2}+o_{\gamma}(1)\left\|\nabla f_{\gamma}\right\|^{2},
$$

with $C_{W}:=\lambda_{0}^{2}\left(1+\lambda_{0} d_{W_{+}}\right) d_{W^{2}}$, where $W_{+}=(W+|W|) / 2$ and $d_{W^{2}}$ and $d_{W_{+}}$are defined by (3) $-($(4) .

Proof. For a potential $V$ such that $V \in L_{\text {loc }}^{2}$ and short range we have

$$
|\langle V \psi, \psi\rangle| \leq d_{V}\|\nabla \psi\|^{2} \text {. }
$$

Moreover, we know that $f_{\gamma}$ is an eigenfunction of $-(1-\gamma) \Delta+\lambda_{0} W$ and that the associated eigenvalue $e_{\gamma}$ tends to zero as $\gamma$ tends to zero, since $\lambda_{0}$ is the critical coupling constant. Therefore we obtain the following sequence of inequalities

$$
\begin{array}{r}
\sum_{i}\left\langle(-\Delta+\lambda W) \frac{\partial f_{\gamma}}{\partial x_{i}}, \frac{\partial f_{\gamma}}{\partial x_{i}}\right\rangle \leq \sum_{i}\left\langle\left(-\Delta+\lambda W_{+}\right) \frac{\partial f_{\gamma}}{\partial x_{i}}, \frac{\partial f_{\gamma}}{\partial x_{i}}\right\rangle \\
\leq \sum_{i}\left\langle\left(-\Delta+\lambda_{0} W_{+}\right) \frac{\partial f_{\gamma}}{\partial x_{i}}, \frac{\partial f_{\gamma}}{\partial x_{i}}\right\rangle \leq \sum_{i}\left\langle-\Delta \frac{\partial f_{\gamma}}{\partial x_{i}}, \frac{\partial f_{\gamma}}{\partial x_{i}}\right\rangle\left(1+d_{\lambda_{0} W_{+}}\right) \\
=\left(1+d_{\lambda_{0} W_{+}}\right)\left\langle-\Delta f_{\gamma},-\Delta f_{\gamma}\right\rangle=\left(1+d_{\lambda_{0} W_{+}}\right)\left\langle\frac{e_{\gamma}-\lambda_{0} W}{1-\gamma} f_{\gamma},-\Delta f_{\gamma}\right\rangle \\
=\frac{e_{\gamma}\left(1+d_{\lambda_{0} W_{+}}\right)}{1-\gamma}\left\langle f_{\gamma},-\Delta f_{\gamma}\right\rangle-\frac{\lambda_{0}\left(1+d_{\lambda_{0} W_{+}}\right)}{1-\gamma}\left\langle W f_{\gamma},-\Delta f_{\gamma}\right\rangle
\end{array}
$$

We estimate the last term in the right hand side by

$$
\begin{aligned}
& -\left\langle W f_{\gamma},-\Delta f_{\gamma}\right\rangle=\left\langle W f_{\gamma}, \frac{\lambda_{0} W-e_{\gamma}}{1-\gamma} f_{\gamma}\right\rangle \\
& \leq-\frac{e_{\gamma} d_{W_{+}}}{1-\gamma}\left\|\nabla f_{\gamma}\right\|^{2}+\frac{\lambda_{0}}{1-\gamma} d_{W^{2}}\left\|\nabla f_{\gamma}\right\|^{2}
\end{aligned}
$$


The Inequalities (20) and (21) imply for $\lambda \leq \lambda_{0}$

$$
\sum_{i}\left\langle(-\Delta+\lambda W) \frac{\partial f_{\gamma}}{\partial x_{i}}, \frac{\partial f_{\gamma}}{\partial x_{i}}\right\rangle \leq\left(\lambda_{0}^{2}\left(1+\lambda_{0} d_{W_{+}}\right) d_{W^{2}}+o_{\gamma}(1)\right)\left\|\nabla f_{\gamma}\right\|^{2}
$$

In the rest of this section, we will mainly work in the space representation for both particle and photons. Following [6], let us introduce, for given $x \in \mathbb{R}^{3}$, the shift operator on the photon space variables $\tau_{x}: \mathbb{C}^{2} \otimes \mathfrak{F} \rightarrow \mathbb{C}^{2} \otimes \mathfrak{F}$. For $\phi=\left(\phi_{0}, \phi_{1}, \ldots, \phi_{n}, \ldots\right) \in \mathbb{C}^{2} \otimes \mathcal{F}$, we have, writing by abuse of notation $\tau_{x} \phi=\left(\tau_{x} \phi_{0}, \tau_{x} \phi_{1}, \ldots\right)$,

$$
\tau_{x} \phi_{n}\left(s ; y_{1}, \ldots, y_{n} ; \lambda_{1}, \ldots, \lambda_{n}\right)=\phi_{n}\left(s ; y_{1}-x, \ldots, y_{n}-x ; \lambda_{1}, \ldots, \lambda_{n}\right),
$$

where $s$ is the spin of the particle and takes value in $\{\uparrow, \downarrow\}$.

We denote by $\Omega_{0}^{x}$ the ground state $\Omega_{0}$ written in space representation and shifted by $x$, i.e.

$$
\Omega_{0}^{x}:=\tau_{x} \mathcal{F}^{-1} \Omega_{0}
$$

where $\mathcal{F}$ stands for the Fourier transform.

Recall that $D^{*}(0)$ is an operator valued vector with 3 components which we denote by $D^{*}(0)_{i}(i=1,2,3)$. Then we consider the functions

$$
\theta_{i}=\left(0, \theta_{i}^{(1)}, 0, \ldots\right) \in \mathbb{C}^{2} \otimes \mathfrak{F}
$$

with

$$
\theta_{i}^{(1)}=\left(k^{2}+|k|+C_{W}\right)^{-1} \Pi_{1} D^{*}(0)_{i}\left(\left(\begin{array}{l}
a \\
b
\end{array}\right), 0, \ldots\right)
$$

and

$$
\theta_{i}^{x}=\tau_{x} \mathcal{F}^{-1} \theta_{i}
$$

We first state some properties of $\theta_{i}$.

Lemma 3.6.

i) For $i \neq j$ we have

$$
\left\langle\theta_{i}, \theta_{j}\right\rangle=0 \quad \text { and } \quad\left\langle\theta_{i}, \theta_{j}\right\rangle_{1}=0 .
$$

ii) For $i=1,2,3$ holds

$$
\left\|\theta_{i} \sqrt{k^{2}+|k|+C_{W}}\right\|^{2}=\frac{1}{6 \pi^{2}} \int_{\mathbb{R}^{3}} \frac{\zeta(|k|)}{|k|\left(k^{2}+|k|+C_{W}\right)} \mathrm{d} k,
$$

iii) For $i=1,2,3,\left\langle k_{i} \varphi_{a, b}, \Pi_{1} \theta_{i}\right\rangle=0$. 
Proof. To prove this Lemma we remind that $\theta_{i}$ has only a non zero component $\Pi_{1} \theta_{i}$ in the one photon sector, and

$$
\Pi_{1} \theta_{i}=\left(\begin{array}{c}
a \frac{\varepsilon_{1, i}(k) \zeta(|k|)}{|k|^{\frac{1}{2}}\left(k^{2}+|k|+C_{W}\right)} \\
a \frac{\varepsilon_{2, i}(k) \zeta(|k|)}{|k|^{\frac{1}{2}}\left(k^{2}+|k|+C_{W}\right)} \\
b \frac{\varepsilon_{1, i}(k) \zeta(|k|)}{|k|^{\frac{1}{2}}\left(k^{2}+|k|+C_{W}\right)} \\
b \frac{\varepsilon_{2, i}(k) \zeta(|k|)}{|k|^{\frac{1}{2}}\left(k^{2}+|k|+C_{W}\right)}
\end{array}\right),
$$

where the two polarization vectors $\varepsilon_{1}(k)$ and $\varepsilon_{2}(k)$ are defined in (2). The properties stated in the Lemma follow straightforwardly from computations of the corresponding integrals.

We consider the trial function $\Psi \in L^{2}\left(\mathbb{R}^{3}\right) \otimes \mathbb{C}^{2} \otimes \mathfrak{F}$ :

$$
\Psi:=\Psi_{1}+\Psi_{2}:=f_{\gamma}(x) \Omega_{0}^{x}+i \sqrt{\alpha} \sum_{i=1}^{3} \theta_{i}^{x} \frac{\partial f_{\gamma}(x)}{\partial x_{i}} .
$$

Now we compute the expectation value of $H$ in the state $\Psi$. We have

$$
\langle H \Psi, \Psi\rangle=\left\langle H \Psi_{1}, \Psi_{1}\right\rangle+\left\langle H \Psi_{2}, \Psi_{2}\right\rangle+2 \mathcal{R} e\left\langle H \Psi_{1}, \Psi_{2}\right\rangle .
$$

As usual [6], due to the orthogonality $\left\langle f, \partial f / \partial x_{i}\right\rangle=0$, we have

$$
\left\langle H \Psi_{1}, \Psi_{1}\right\rangle=\Sigma_{0}\left\|\Psi_{1}\right\|^{2}+\left\langle(-\Delta+\lambda W(x)) f_{\gamma}, f_{\gamma}\right\rangle\left\|\Omega_{0}\right\|^{2}
$$

Since $\Psi_{2}$ has only a non zero component in the one photon sector, in the quadratic form $\left\langle H \Psi_{2}, \Psi_{2}\right\rangle$, all the terms involving $A(0)$ or $B(0)$ vanish. Moreover, using Lemma 3.6] and the orthogonalities $\left\langle\frac{\partial f_{\gamma}}{\partial x_{i}}, \frac{\partial f_{\gamma}}{\partial x_{j}}\right\rangle=0$ and $\left\langle\frac{\partial f_{\gamma}}{\partial x_{i}}, \frac{\partial^{2} f_{\gamma}}{\partial x_{i} \partial x_{j}}\right\rangle=0$, for $i \neq j$, we arrive at

$$
\begin{aligned}
\left\langle H \Psi_{2}, \Psi_{2}\right\rangle= & \alpha \sum_{l}\left\|\theta_{l}^{x}\right\|^{2}\left\langle(-\Delta+\lambda W) \frac{\partial f_{\gamma}}{\partial x_{l}}, \frac{\partial f_{\gamma}}{\partial x_{l}}\right\rangle+\mathcal{O}\left(\alpha^{2}\right)\left\|\nabla f_{\gamma}\right\|^{2} \\
& +\alpha \sum_{l}\left\|\frac{\partial f_{\gamma}}{\partial x_{l}}\right\|^{2}\left\langle\left(|k|+k^{2}\right) \Pi_{1} \theta_{l}^{x}, \Pi_{1} \theta_{l}^{x}\right\rangle .
\end{aligned}
$$

To compute the last term $\left\langle H \Psi_{1}, \Psi_{2}\right\rangle$, we first note that

$$
\begin{aligned}
\left\langle(-\Delta+\lambda W) f_{\gamma}, \frac{\partial f_{\gamma}}{\partial x_{i}}\right\rangle & =\left\langle(-(1-\gamma) \Delta+\lambda W) f_{\gamma}, \frac{\partial f_{\gamma}}{\partial x_{i}}\right\rangle-\gamma\left\langle\Delta f_{\gamma}, \frac{\partial f_{\gamma}}{\partial x_{i}}\right\rangle \\
& =e_{\gamma}\left\langle f_{\gamma}, \frac{\partial f_{\gamma}}{\partial x_{i}}\right\rangle+\gamma \sum_{j}\left\langle\frac{\partial^{2} f_{\gamma}}{\partial x_{j}^{2}}, \frac{\partial f_{\gamma}}{\partial x_{i}}\right\rangle=0 .
\end{aligned}
$$


The last equality holds since $f_{\gamma}$ is a real function vanishing at infinity. Moreover, all other terms in the quadratic form $\left\langle H \Psi_{1}, \Psi_{2}\right\rangle$ which contain $\left\langle f_{\gamma}, \frac{\partial f_{\gamma}}{\partial x_{i}}\right\rangle$ vanish also. So we arrive at

$$
\begin{aligned}
2 \mathcal{R} e\left\langle H \Psi_{1}, \Psi_{2}\right\rangle & =-2 \mathcal{R} e\left\langle P \cdot\left(P_{f}-\sqrt{\alpha} A(0)\right) \Psi_{1}, \Psi_{2}\right\rangle \\
& =2 \sqrt{\alpha} \sum_{i}\left\|\frac{\partial f_{\gamma}}{\partial x_{i}}\right\|^{2} \mathcal{R} e\left\langle\left(P_{f}-\sqrt{\alpha} A(0)\right)_{i} \Omega_{0}, \theta_{i}\right\rangle
\end{aligned}
$$

The term with $P_{f}$ on the right hand side is estimated as follows

$$
\begin{aligned}
\mathcal{R} e\left\langle\left(P_{f}\right)_{i} \Omega_{0}, \theta_{i}\right\rangle_{\mathbb{C}^{2} \otimes \mathfrak{F}} & =\mathcal{R} e\left\langle\Pi_{1}\left(P_{f}\right)_{i}\left(\gamma \varphi_{a, b}+R\right), \Pi_{1} \theta_{i}\right\rangle_{\mathbb{C}^{2} \otimes L^{2}\left(\mathbb{R}^{3}\right) \otimes \mathbb{C}^{2}} \\
& =\mathcal{R} e\left\langle k_{i}\left(\gamma \varphi_{a, b}+R\right), \Pi_{1} \theta_{i}\right\rangle_{\mathbb{C}^{2} \otimes L^{2}\left(\mathbb{R}^{3}\right) \otimes \mathbb{C}^{2}} \\
& \leq\|R\|_{1}\left\||k|^{\frac{1}{2}} \Pi_{1} \theta_{i}\right\|+\gamma \mathcal{R} e\left\langle k_{i} \varphi_{a, b}, \Pi_{1} \theta_{i}\right\rangle_{\mathbb{C}^{2} \otimes L^{2}\left(\mathbb{R}^{3}\right) \otimes \mathbb{C}^{2}}
\end{aligned}
$$

Using Proposition 3.3 yields the following bound for the first term in the right hand side of (30)

$$
\|R\|_{1}\left\||k|^{\frac{1}{2}} \Pi_{1} \theta_{i}\right\|=\mathcal{O}\left(\alpha^{\frac{3}{4}}\right)
$$

According to Lemma 3.6 iii), the second term in the right hand side of (30) equals zero. Therefore, collecting (29)-(31), we arrive at

$$
2 \mathcal{R} e\left\langle H \Psi_{1}, \Psi_{2}\right\rangle=-2 \alpha \sum_{i}\left\|\frac{\partial f_{\gamma}}{\partial x_{i}}\right\|^{2} \mathcal{R} e\left\langle A(0)_{i} \Omega_{0}, \theta_{i}\right\rangle+\mathcal{O}\left(\alpha^{\frac{5}{4}}\right)\left\|\nabla f_{\gamma}\right\|^{2}
$$

Now we have, using Theorem 3.1 and the fact that $D(0)$ restricted to the 2-photon sector is a bounded operator

$$
\begin{aligned}
\mathcal{R} e\left\langle A(0)_{i} \Omega_{0}, \theta_{i}\right\rangle= & \mathcal{R} e\left\langle D(0)_{i} \Pi_{2} \Omega_{0}, \theta_{i}\right\rangle+\mathcal{R} e\left\langle D^{*}(0)_{i} \Pi_{0} \Omega_{0}, \theta_{i}\right\rangle \\
& =\mathcal{O}\left(\alpha^{\frac{1}{2}}\right)+\mathcal{R} e\left\langle D^{*}(0)_{i} \Pi_{0} \Omega_{0}, \theta_{i}\right\rangle
\end{aligned}
$$

Due to the definition (24) of $\theta_{i}$, the second term on the right hand side of (33) is $\left\|\theta_{i} \sqrt{k^{2}+|k|+C_{W}}\right\|^{2}$. Therefore, collecting the Equalities (27), 
(28), (32) and (33) we obtain

$$
\begin{aligned}
\langle H \Psi, \Psi\rangle= & \Sigma_{0}\left\|\Psi_{1}\right\|^{2}+\left\|\Omega_{0}\right\|^{2}\left\langle(-\Delta+\lambda W) f_{\gamma}, f_{\gamma}\right\rangle \\
& +\alpha \sum_{l}\left\|\theta_{l}\right\|^{2}\left\langle(-\Delta+\lambda W) \frac{\partial f_{\gamma}}{\partial x_{l}}, \frac{\partial f_{\gamma}}{\partial x_{l}}\right\rangle \\
& -2 \alpha \sum_{l}\left\|\frac{\partial f_{\gamma}}{\partial x_{l}}\right\|^{2}\left\|\theta_{i} \sqrt{k^{2}+|k|+C_{W}}\right\|^{2}+\mathcal{O}\left(\alpha^{\frac{5}{4}}\right)\left\|\nabla f_{\gamma}\right\|^{2} \\
& +\alpha \sum_{l}\left\|\frac{\partial f_{\gamma}}{\partial x_{l}}\right\|^{2}\left\|\theta_{l}\right\|_{1}^{2} .
\end{aligned}
$$

From Lemma 3.6. we know that $\left\|\theta_{l} \sqrt{k^{2}+|k|+C_{W}}\right\|^{2}$ is independent of $l$. We denote this constant by $\eta^{2}$. With Lemma 3.5 we thus arrive at

$$
\begin{aligned}
\langle H \Psi, \Psi\rangle \leq & \Sigma_{0}\|\Psi\|^{2}-\Sigma_{0}\left\|\Psi_{2}\right\|^{2}+\left\|\Omega_{0}\right\|^{2}\left(\left\|\nabla f_{\gamma}\right\|^{2}+\left\langle\lambda W f_{\gamma}, f_{\gamma}\right\rangle\right) \\
& -\alpha \sum_{l}\left\|\frac{\partial f_{\gamma}}{\partial x_{l}}\right\|^{2} \eta^{2}+\alpha o_{\gamma}(1)\left\|\nabla f_{\gamma}\right\|^{2}+\mathcal{O}\left(\alpha^{\frac{5}{4}}\right)\left\|\nabla f_{\gamma}\right\|^{2} .
\end{aligned}
$$

Note that $\Sigma_{0}\left\|\psi_{2}\right\|^{2}=\mathcal{O}\left(\alpha^{2}\right)\left\|\nabla f_{\gamma}\right\|^{2}$. We thus obtain

$$
\begin{aligned}
& \langle H \Psi, \Psi\rangle-\Sigma_{0}\|\Psi\|^{2} \leq \\
& \left\|\Omega_{0}\right\|^{2}\left(\left(1-\frac{\alpha}{\left\|\Omega_{0}\right\|^{2}} \eta^{2}+\frac{\alpha}{\left\|\Omega_{0}\right\|^{2}}\left(o_{\gamma}(1)+\mathcal{O}\left(\alpha^{\frac{1}{4}}\right)\right)\right)\left\|\nabla f_{\gamma}\right\|^{2}+\left\langle\lambda W f_{\gamma}, f_{\gamma}\right\rangle\right)
\end{aligned}
$$

Using from Corollary 3.2 that $\left\|\Omega_{0}\right\|^{2}=1+\mathcal{O}(\alpha)$, we obtain

$$
\begin{array}{r}
\langle H \Psi, \Psi\rangle-\Sigma_{0}\|\Psi\|^{2} \leq \\
\left\|\Omega_{0}\right\|^{2}\left(\left(1-\alpha \eta^{2}+\alpha o_{\gamma}(1)+\mathcal{O}\left(\alpha^{\frac{5}{4}}\right)\right)\left\|\nabla f_{\gamma}\right\|^{2}+\left\langle\lambda W f_{\gamma}, f_{\gamma}\right\rangle\right) .
\end{array}
$$

Therefore

$$
\begin{aligned}
& \langle H \Psi, \Psi\rangle-\Sigma_{0}\|\Psi\|^{2} \leq\left\|\Omega_{0}\right\|^{2}\left(1-\alpha \eta^{2}+\alpha o_{\gamma}(1)+\mathcal{O}\left(\alpha^{\frac{5}{4}}\right)\right) \\
\times & \left(\left\|\nabla f_{\gamma}\right\|^{2}+\left(1+\alpha \eta^{2}+\alpha o_{\gamma}(1)+\mathcal{O}\left(\alpha^{\frac{5}{4}}\right)\right)^{-1}\left\langle\lambda W f_{\gamma}, f_{\gamma}\right\rangle\right) .
\end{aligned}
$$

If $\lambda>\lambda_{0}\left(1-\alpha \eta^{2}+\mathcal{O}\left(\alpha^{\frac{5}{4}}\right)\right)$, choosing $\gamma$ (depending on $\alpha$ ) small enough, we arrive at $\langle H \Psi, \Psi\rangle-\Sigma_{0}\|\Psi\|^{2}<0$. Due to Griesemer et al. [8], this implies the existence of a ground state for $H$.

\section{REFERENCES}

[1] J.-M. Barbaroux, T. Chen, S.A. Vugalter, Binding conditions for atomic $N$ particle systems in non-relativistic QED, Ann. Henri Poincaré, 4: 1101-1136, 2003. 
[2] R.D. Benguria, S.A. Vugalter, Binding threshold for the Pauli-Fierz operator, Lett. Math. Phys., 70: 249-257, 2004.

[3] I. Catto, C. Hainzl, Self-energy of one electron in non-relativistic QED, J. Funct. Anal., 207: 68-110, 2004.

[4] I. Catto, P. Exner, C. Hainzl, Enhanced binding revisited for a spinless particle in nonrelativistic QED, J. Math. Phys., 45: 4174-4185, 2004.

[5] T. Chen, Operator-theoretic infrared renormalization and construction of dressed 1-electron states in non-relativistic QED, preprint arXiv: math-ph/0108021, 2001.

[6] T. Chen, V. Vougalter, S. A. Vugalter, The increase of binding energy and enhanced binding in non-relativistic QED, J. Math. Phys., 44 (5), 2003.

[7] J. Fröhlich, Existence of dressed one electron states in a class of persistent models. Fortschritte der Physik, 22: 159-189, 1974.

[8] M. Griesemer, E. H. Lieb, M. Loss, Ground states in non-relativistic quantum electrodnamics, Inv. Math, 145: 557-595, 2001.

[9] C. Hainzl, V. Vougalter, S.A. Vugalter, Enhanced binding in non-relativistic QED, Comm. Math. Phys., 233: 13-26, 2003.

[10] F. Hiroshima, H. Spohn, Enhanced binding through coupling to a quantum field, Ann. Henri Poincar 2, no. 6: 1159-1187, 2001.

Centre de Physique Théorique, Luminy Case 907, 13288 Marseille Cedex 9, France and Département de Mathématiques, Université du Sud-Toulon-Var, avenue de l'Université, 83957 la Garde Cedex, France, BARBAROU@UNIV-TLN.FR

Facultad de Fìsica, P. Universidad Católica de Chile, Casilla 306, Santiago 22, Chile

Mathematisches Institut, Ludwig-Maximilians-Universität MÜNChen, Theresienstrasse 39, 80333 MÜnChen and Institut für Analysis, DyNAmik und Modelierung, Universität Stuttgart, wUGalter@Mathematik.uniMUENCHEN.DE 